\title{
First Imaging of Coronal Mass Ejections in the Heliosphere Viewed from Outside the Sun-Earth Line
}

\author{
Richard A. Harrison - Christopher J. Davis • Christopher J. Eyles • Danielle Bewsher • \\ Steve R. Crothers • Jackie A. Davies • Russell A. Howard • Daniel J. Moses • \\ Dennis G. Socker · Jeffrey S. Newmark · Jean-Philippe Halain • Jean-Marc Defise • \\ Emmanuel Mazy • Pierre Rochus • David F. Webb • George M. Simnett
}

Received: 25 June 2007 / Accepted: 31 October 2007

(C) Springer Science+Business Media B.V. 2007

\begin{abstract}
We show for the first time images of solar coronal mass ejections (CMEs) viewed using the Heliospheric Imager (HI) instrument aboard the NASA STEREO spacecraft. The $\mathrm{HI}$ instruments are wide-angle imaging systems designed to detect CMEs in the heliosphere, in particular, for the first time, observing the propagation of such events along the SunEarth line, that is, those directed towards Earth. At the time of writing the STEREO spacecraft are still close to the Earth and the full advantage of the HI dual-imaging has yet to be realised. However, even these early results show that despite severe technical challenges in their design and implementation, the HI instruments can successfully detect CMEs in the heliosphere, and this is an extremely important milestone for CME research. For the principal event being analysed here we demonstrate an ability to track a CME from the corona to over 40 degrees. The time - altitude history shows a constant speed of ascent over at least the first 50 solar radii and some evidence for deceleration at distances of over 20 degrees.
\end{abstract}

\footnotetext{
R.A. Harrison $(\bowtie) \cdot$ C.J. Davis · C.J. Eyles · D. Bewsher · S.R. Crothers · J.A. Davies Space Science and Technology Department, Rutherford Appleton Laboratory, Chilton, Didcot, Oxfordshire OX11 0QX, UK

e-mail: r.harrison@rl.ac.uk

C.J. Eyles · G.M. Simnett

School of Physics and Astronomy, University of Birmingham, Birmingham B15 2TT, UK

C.J. Eyles

Grupo de Astronomia y Ciencias del Espacio, ICMUV, Universidad de Valencia, Valencia, Spain

R.A. Howard · D.J. Moses · D.G. Socker · J.S. Newmark

Space Science Division, Naval Research Laboratory, Washington, DC, USA

J.-P. Halain · J.-M. Defise · E. Mazy · P. Rochus

Centre Spatial de Liège, Université de Liège, ave Pré Aily, 4031 Angleur, Belgium

D.F. Webb

Institute for Scientific Research, Boston College, Chestnut Hill, MA, USA

D.F. Webb

Air Force Research Laboratory, Hanscom, AFB, MA, USA
} 
Comparisons of associated coronagraph data and the HI images show that the basic structure of the CME remains clearly intact as it propagates from the corona into the heliosphere. Extracting the CME signal requires a consideration of the F-coronal intensity distribution, which can be identified from the HI data. Thus we present the preliminary results on this measured F-coronal intensity and compare these to the modelled F-corona of Koutchmy and Lamy (IAU Colloq. 85, 63, 1985). This analysis demonstrates that CME material some two orders of magnitude weaker than the F-corona can be detected; a specific example at 40 solar radii revealed CME intensities as low as $1.7 \times 10^{-14}$ of the solar brightness. These observations herald a new era in CME research as we extend our capability for tracking, in particular, Earth-directed CMEs into the heliosphere.

Keywords Coronal mass ejection $\cdot$ Heliosphere

\section{Introduction}

The propagation of coronal mass ejections (CMEs) along the Sun-Earth line is a topic of significant interest to the solar and solar-terrestrial physics communities. CMEs are usually detected using coronagraphs on platforms either in Earth orbit or near to the Earth (e.g., an L1 orbit). Such instruments, being best suited to the detection of ejecta in the plane of the sky, are capable of detecting Earth-directed CMEs as halo CMEs (Howard et al., 1982), but these halo events are often rather weak in intensity and we probably miss many. Even when a halo is detected, this provides little information on its propagation along the Sun -Earth line and on the development of a CME as it approaches Earth. Ideally, one would wish to view the Sun -Earth line with a wide-angle imaging system from a vantage point well away from the Sun - Earth line.

We have been careful to talk of CMEs and interplanetary coronal mass ejections (ICMEs) over the years, loosely defining the former as ejecta detected close to the Sun using coronagraph instrumentation and the latter as ejecta usually detected using in situ particle and field measurements in interplanetary space. The precise relationship between the two is unclear but it is generally accepted that an ICME is the interplanetary counterpart of a CME. To illustrate this we refer the reader to Riley et al. (2006), who showed that through the period 1996 to 2004 the CME and ICME rates are consistent with one another, and to the papers of Gopalswamy et al. (2000, 2001), where comparisons of individual events were listed for observations from a range of spacecraft. However, studies such as these usually involve the comparison of data taken at two locations, near the Sun and near the Earth, and understanding how such events propagate between the Sun and Earth has been a topic of great interest. Gopalswamy et al. (2000) measured the speeds of CMEs at the Sun and, comparing them to in situ signatures near the Earth, produced an empirical model for the interplanetary trajectory of CMEs. They refined the model significantly (Gopalswamy et al., 2001) by using data from spacecraft widely spaced in the inner Solar System, recognising that for halo CMEs the apparent speeds measured were not necessarily the speeds of the CME front. As a result, for 19 events they produced clear associations between CMEs and ICMEs and these were used to produce an improved empirical model for CME speed profiles in transit from the Sun to Earth. To obtain the best results, they introduced a cessation of acceleration, with the best match at 0.76 AU. Gonzalez-Esparza et al. (2003) took this further with a numerical model in which the CME is expanding into different solar wind backgrounds. Clearly, the modelled CME and solar wind plasma interact, bringing the CME speed nearer to the solar wind flow. Fast CMEs (faster than the ambient solar wind) showed rapid deceleration at less 
than $0.1 \mathrm{AU}$, followed by a period of constant speed for several tenths of an AU, then further, gradual deceleration. In contrast, slow CMEs showed rapid initial acceleration, within 0.1 AU and pretty constant outflows to $1 \mathrm{AU}$, with some gradual deceleration.

Direct observations of CMEs in the heliosphere between the Sun and Earth, over long periods (i.e., not just comparisons at $1 \mathrm{AU}$ and the Sun), would clearly be of benefit for predicting the arrival of CMEs at Earth and for testing, and perhaps refining, these models of CME speed profiles. Indeed, the very nature of the projections and empirical models we mention stresses the fact that we have been limited to observations effectively at only two points in space.

The fact that we usually detect CMEs through near-Sun remote sensing, and ICMEs through in situ particle and field sampling, results in additional dilemmas in relating the two. CMEs are characterised by their speeds, acceleration, sizes, and masses but we lack basic plasma and field parameters; the magnetic field strength and direction are not known, nor are plasma parameters such as charge-state composition and elemental abundances, yet these are the very parameters that we can measure in situ for ICMEs. A recent review of ICME signatures was given by Wimmer-Schweingruber et al. (2006) and this included case studies such as that of the so-called Bastille Day CME of 14 July 2000. However, the review of this event serves to stress that the halo CME discussion and associated solar activity are rather disconnected from the discussion of the associated ICME. It is clear that with an ability to track the CME through the heliosphere we would have a far better understanding of the time of arrival, the mass, and structure of the event as it passes over the Earth, including some knowledge of how the Earth cuts through the CME structure.

This last point is important. Over the years, much has been made of the fact that there is a "typical" three-part structure to CMEs close to the Sun and it is often very difficult to match in situ signatures to such a structure. Wimmer-Schweingruber et al. (2006) review some of the relevant work and discuss the issue of boundaries within ICMEs and the 3D structure of CMEs. If one considers the dense leading edge, followed by void, including cold prominence material, as the basic three-part CME structure, we can interpret a compressed ICME front and the ensuing ICME as counterparts of the first two, and the occasional identification of cool, dense material as the associated prominence. However, there is considerable eventto-event variation. This will be due to the variation in CME structure and topology, and, as already noted, where in that structure the in situ measurements are made. An imaging capability well away from the Sun-Earth line is thus required to interpret in situ ICME observations in the vicinity of the Earth.

Perhaps most significantly, Wimmer-Schweingruber et al. (2006) concluded that the identification of ICMEs in situ is not straightforward because there is no single signature or combination of signatures that is foolproof for the identification of an ICME. This stresses again that the key to understanding and even identifying ICMEs is through the tracking of CMEs to the vicinity of the Earth utilising imaging techniques.

It is not the purpose of this paper to provide a complete review of the CME and ICME literature. The purpose of this paper is to announce a new chapter in CME and ICME research and to demonstrate a new imaging capability. The discussion here illustrates some of the outstanding issues that will be addressed by this capability. For recent detailed reviews we refer the reader to the many papers in the conference proceedings of Gopalswamy, Mewaldt, and Torsti (2006) and Kunow et al. (2006). 


\section{Relevant Space Instrumentation}

In the mid to late 1970s the Helios spacecraft operated from solar orbits with a perihelion of $0.31 \mathrm{AU}$, and zodiacal light photometers were used to detect CMEs (see, e.g., Richter, Leinert, and Planck, 1982; Jackson and Leinert, 1985). The CME images were constructed from three photometer directions that scanned the sky using the rotation of the spacecraft; this resulted in spatial coverage from which CME structures could be mapped. This technique required considerable interpretation and projection but it is clear that CMEs were detected successfully out of the Sun-Earth line. A recent major advance was made with the launch of the Solar Mass Ejection Imager (SMEI) aboard the Coriolis spacecraft, in 2003 (Eyles et $a l ., 2003)$. This instrument maps the entire sky with three cameras, each scanning 60-degree slices of the sky as the spacecraft moves around the Earth. This instrument has pioneered full-sky mapping aimed specifically at the detection of CMEs propagating through the inner heliosphere but, of course, it does not view CMEs from out of the Sun-Earth line.

The combination of wide-angle heliospheric mapping from out of the Sun - Earth line is now being satisfied by the Heliospheric Imagers (HI) aboard the NASA STEREO spacecraft. Hence, these instruments are unique in their ability to image those events directed towards the Earth. Indeed, for the first time, the HI instruments provide a view of the passage of CMEs along virtually the entire Sun-Earth line and such observations represent a major milestone in investigations of the influence of solar activity on the Earth and human systems.

The HI instruments, carried aboard each of the pair of STEREO spacecraft, are part of the SECCHI package of remote sensing instruments designed to study the solar disc, the corona, and heliosphere, from the Sun's chromosphere to the Earth and beyond. The HI instruments and their operation are described in detail by Socker et al. (2000), Harrison, Davis, and Eyles (2005), Defise et al. (2003), and Davis and Harrison (2005), and the SECCHI package is described by Howard, Moses, and Socker (2000).

\section{The Heliospheric Imagers}

The STEREO spacecraft were launched at 01:52 UT on 26 October 2006 and inserted into near 1 AU solar orbits, one leading and one lagging the Earth after lunar swing-bys in December and January. The leading spacecraft is labelled STEREO A (Ahead) and the lagging spacecraft, STEREO B (Behind). The basic orbital configuration is such that the spacecraft recede from the Earth by 22.5 degrees per year, as measured by the spacecraft - Sun -Earth angle. The wide spacecraft-Sun-spacecraft angle, achieved as the mission matures, enables the production of 3D images, from the solar disc-viewing instruments. Moreover, for the HI instruments, the unique orbits also allow the uninterrupted view of the Sun-Earth line from 4 to 88.7 degrees from Sun centre, enabling the study of Earth-directed CMEs and providing the potential for 3D studies of CME structure and evolution.

After commissioning activities, the $\mathrm{HI}$ instrument doors were opened on spacecraft $\mathrm{A}$ on 13 December 2006 and on spacecraft B on 11 January 2007. After operational tests, including image calibration using stellar sources, and checks on image handling and processing software, the HI instruments started their science operations phase in April 2007.

The basic geometrical and operational characteristics of the instruments are shown in Table 1 and Figures 1 and 2. Each HI instrument comprises two refractive telescopes, known as HI-1 and HI-2. Using the labels A and B for the Ahead and Behind spacecraft, respectively, we have four telescopes, which we denote HI-1A, HI-2A, HI-1B, and HI-2B. Both HI-1 telescopes are identical and both HI-2 telescopes are identical. We note that although 
Table 1 Basic parameters of the HI telescopes.

\begin{tabular}{lll}
\hline & HI-1 & HI-2 \\
\hline Direction of centre of field of view from Sun centre & 14 degrees & 53.7 degrees \\
Angular field of view & 20 degrees & 70 degrees \\
Angular range & $4-24$ degrees & $18.7-88.7$ degrees \\
Image array $(2 \times 2$ binning) & $1024 \times 1024$ & $1024 \times 1024$ \\
Image pixel size & $70 \operatorname{arcsec}$ & 4 arcmin \\
Band pass & $630-730 \mathrm{~nm}$ & $400-1000 \mathrm{~nm}$ \\
\hline
\end{tabular}

Figure 1 The fields of view of the HI telescopes and the SOHO/LASCO C3 instrument.

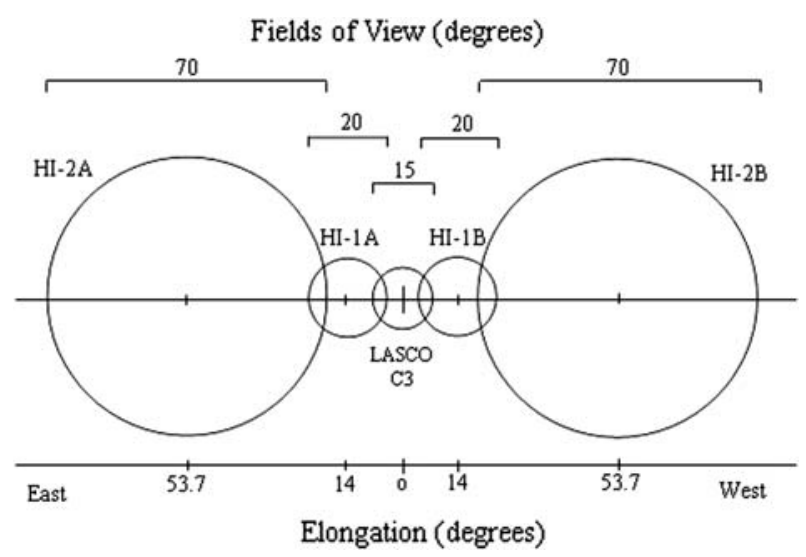

$2048 \times 2048$ CCD pixel detector arrays are used, in standard operation, the images are $2 \times 2$ binned onboard to give $1024 \times 1024$ images.

Figure 1 shows the geometrical layout of the HI telescopes relative to the Sun and to the SOHO LASCO C3 coronagraph (Brueckner et al., 1995). This diagram serves to stress the scale of the new imaging capability compared to the coronagraph images to which we have become accustomed and shows how much of the inner heliosphere is covered by the HI instruments. The abscissa is elongation, both east and west, with the Sun at zero elongation, at the centre of the C3 15-degree field. The central elongation angles of the HI telescopes and their fields of view are identified. It must be noted that this is shown as one flat diagram, for illustration, yet the spacecraft are not co-located but widely spaced and separating with time. The HI-A and HI-B fields will in fact cover common areas of the heliosphere when the spacecraft are well separated.

Figure 2, adapted from Socker et al. (2000), again shows the geometrical layout of the HI fields of view but with a schematic of the coronal and CME intensity contributions. Figures 1 and 2 reveal the overlap between the HI- 1 and the HI- 2 fields of view of 5.3 degrees, which allows a photometric cross-calibration of the images to be made.

To enable efficient pointing of the high-gain antenna, in the early phase of the operation, the geometry is such that the HI fields of view are tilted out of the ecliptic plane. Thus, whereas the axis of the HI and C3 fields of view, as shown in Figures 1 and 2, is nominally the ecliptic plane, in the early stages of the mission, this axis is tilted with respect to the ecliptic plane. This is discussed in the following for the images presented.

During the mission, the Earth appears to move along the ecliptic plane and at times is visible in either or both of the HI fields of view in spacecraft A and B. 
Figure 2 The field of view geometry of the HI telescopes and the anticipated intensities of the corona and CMEs, adapted from Socker et al. (2000).

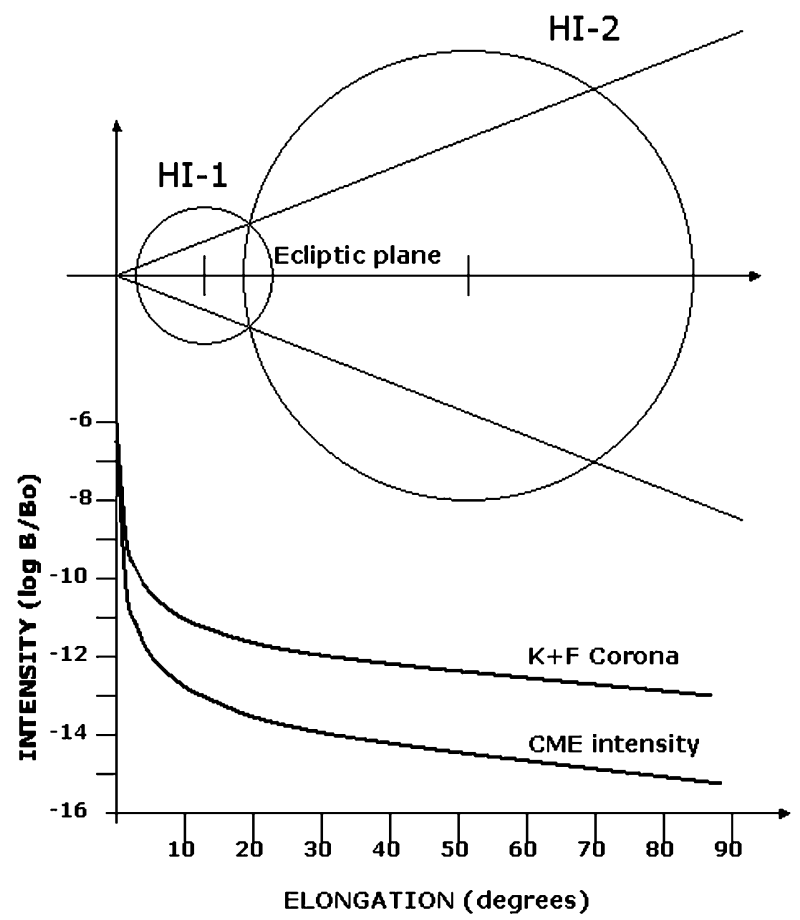

The lower panel of Figure 2 shows, on the same elongation axis, the anticipated signal of the $\mathrm{F}+\mathrm{K}$ corona, in units of $B_{\mathrm{o}}$, the solar disc intensity. This curve is based on projections calculated by Koutchmy and Lamy (1985). Also shown is the anticipated intensity of an average $\mathrm{CME}$, as a function of elongation. The CME intensity is thought to be typically some two orders of magnitude weaker than the F-coronal intensity in the heliosphere. To extract the CME signal one must make long-duration exposures so that the statistical noise in the measurement of the F-coronal signal is much less than the CME signal; one can then use subtraction techniques to isolate the CME.

In practice, the long-duration exposures are made up of many shorter exposures, each cleaned onboard to remove the ionisation tracks in the CCDs resulting from the passage of cosmic rays prior to being summed; a single long-duration exposure would be swamped by cosmic rays. These techniques are discussed in the $\mathrm{HI}$ instrument references given previously.

It is worth noting that in isolating a CME near 4 degrees from Sun centre we are requiring the identification of a source of intensity at best $10^{-12}$ of that of the solar disc, and this is a challenge in terms of eliminating scattered light from the Sun as well as in extracting the signal from the background F-coronal intensity. It is the primary purpose of this early paper to show that this has indeed been achieved.

We describe a sequence of events in detail later, but first we show a selection of images that demonstrate graphically the performance of the HI instruments.

Figure 3 is an HI-1 image that shows one of the first CMEs to be detected using the STEREO instrumentation. This particular event will be discussed in some detail in the following section. However, to put this image in context, the reader should refer to the layout shown in Figure 1. We note here, also, that whilst the prime optical fields of view of both the 
Figure 3 An HI-1A image taken on 25 January 2007. This image is 20 degrees across ( 80 solar radii) and the Sun is 4 degrees to the right of the frame. The planets Venus and Mercury can be seen (lower left and right, respectively) and a CME is clearly expanding into the right-hand side of the image.

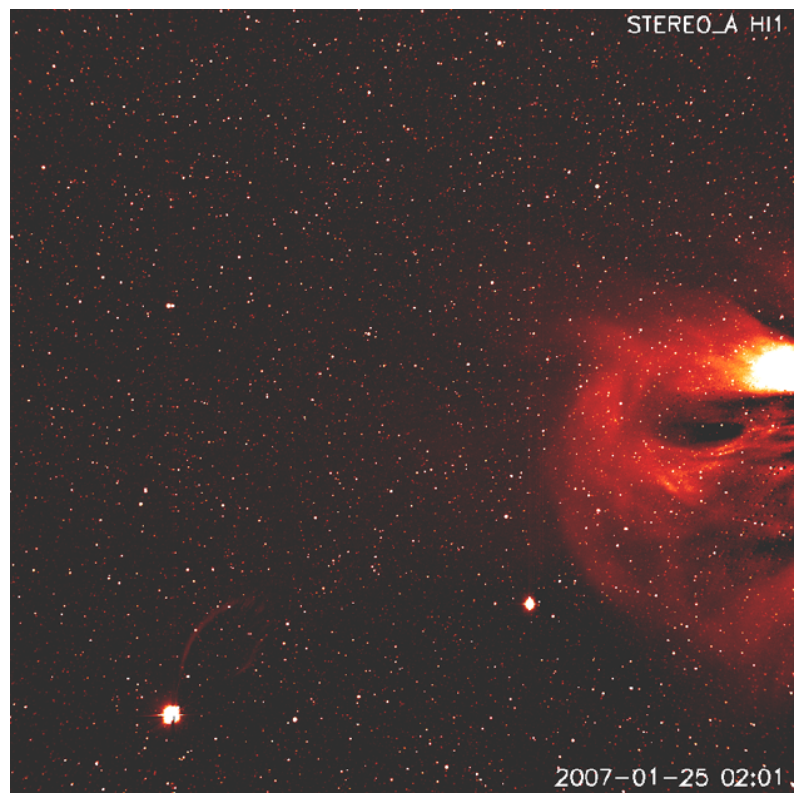

HI-1 and HI-2 instruments are circular, the detector arrays are square and useful information, albeit with somewhat degraded performance, can be obtained in the corners of the detector image, especially for the HI-1 images. For HI-2, we actually image the baffle system in the corners of the field. Thus, we show the HI-1 region as square.

A panorama of the complete HI imaging system, from both spacecraft, is shown in Figure 4, though the quality of the image is somewhat compromised by the need to compress the images into one composite display of limited size. The resolution has been degraded to produce this display. The Sun is shown approximately to scale in Figure 4, between the two HI-1 frames. To ease the display the 4-degree gap between the Sun and HI-1 inner edge has been reduced (see Figures 1 and 2 for reference).

One feature we would draw attention to is the location of the Earth within the HI-1B image. A ghost is evident (the ring feature to the right of the Earth) owing to the brightness of the Earth, but it is clear that the extreme intensity of the Earth does not mask or wipe out useful observations in that part of the HI system in which it appears, even this early in the mission.

Another interesting feature is Comet McNaught, which has passed perihelion and is receding. Fulle et al. (2007) presented HI observations of this comet.

Figure 5 shows a larger format combined HI-1 and HI-2 image from STEREO A for the same period as the image shown in Figure 3. We remind the reader that the (square) HI-1 field of view is 20 degrees across and the larger HI-2 field of view is 70 degrees across. The 5.3-degree overlap between the fields is evident. The stellar intensities in these frames are a measure of the quality of these images and preliminary measurements suggest that we are detecting sources down to magnitude 13. However, expanding CMEs also weaken in intensity as they propagate outwards, so the challenge for extracting CME signals in the HI-2 fields is severe. We show the 25 January 2007 CME in the HI-2A field of view, in Figure 6, using a differenced image.

A more complete description of the 25 January event is given later, but Figure 6 clearly demonstrates the capability for detecting CMEs in the HI-2 field. 


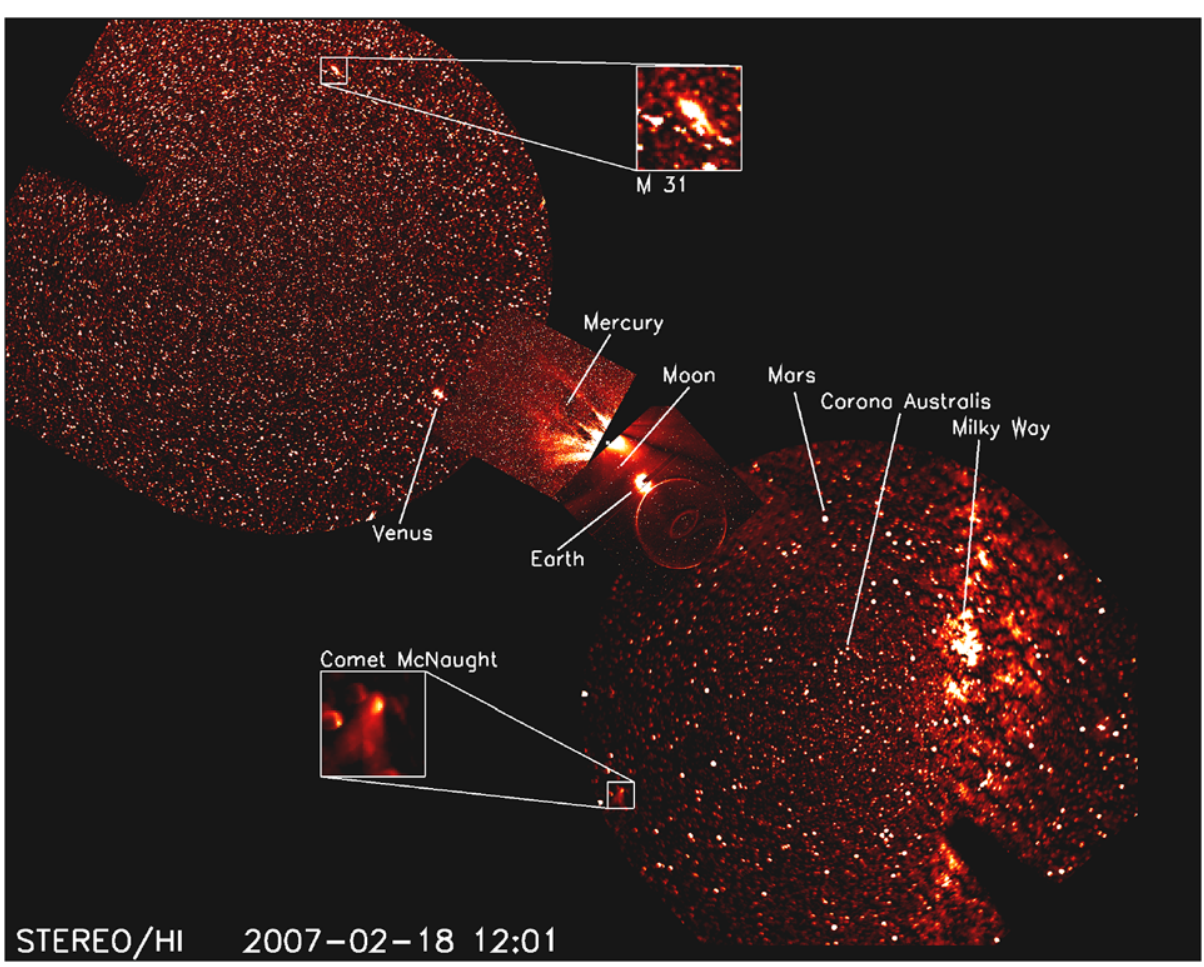

Figure 4 A composite of all HI images (refer to Figure 1 for the format) taken on 18 February 2007. The Sun is shown approximately to scale, between the HI-1 frames, and a number of planetary and astronomical sources are identified. This composite image necessarily compromises the display of the true image resolutions but does stress the heliospheric coverage.

As already mentioned, individual exposures that make up an $\mathrm{HI}$ image are cleaned onboard to remove the effects of cosmic rays before summing and transmitting to Earth. The returned images must then undergo routine processing to remove instrumental effects. One important effect on the images results from the fact that the HI instruments do not have shutters. The CCD read-out and clear-out process means that, in addition to the "prime" exposure, each exposure includes an extra component, which is, in effect, a string of miniexposures as the image is transferred down the CCD columns during the image read-out and clear-out. This results in a smearing effect that can be corrected for on the ground. This correction has been applied for all images shown in this paper. In addition, to extract CME intensities, we need first to remove the F-coronal intensities. This can be done via a number of methods, including the subtraction of successive images (to reveal transient activity between frames), by subtracting a modelled F-coronal distribution, or by subtracting an F-coronal distribution that is established from analysis of minimum intensities in frames around the time of the observation. The F-corona has been removed from all frames in this paper. When this is done, we find very little scattered sunlight and have the ability to detect discrete stellar sources down to at least 13th magnitude. All of these processes are described in detail in an $\mathrm{HI}$ instrument paper in preparation. 


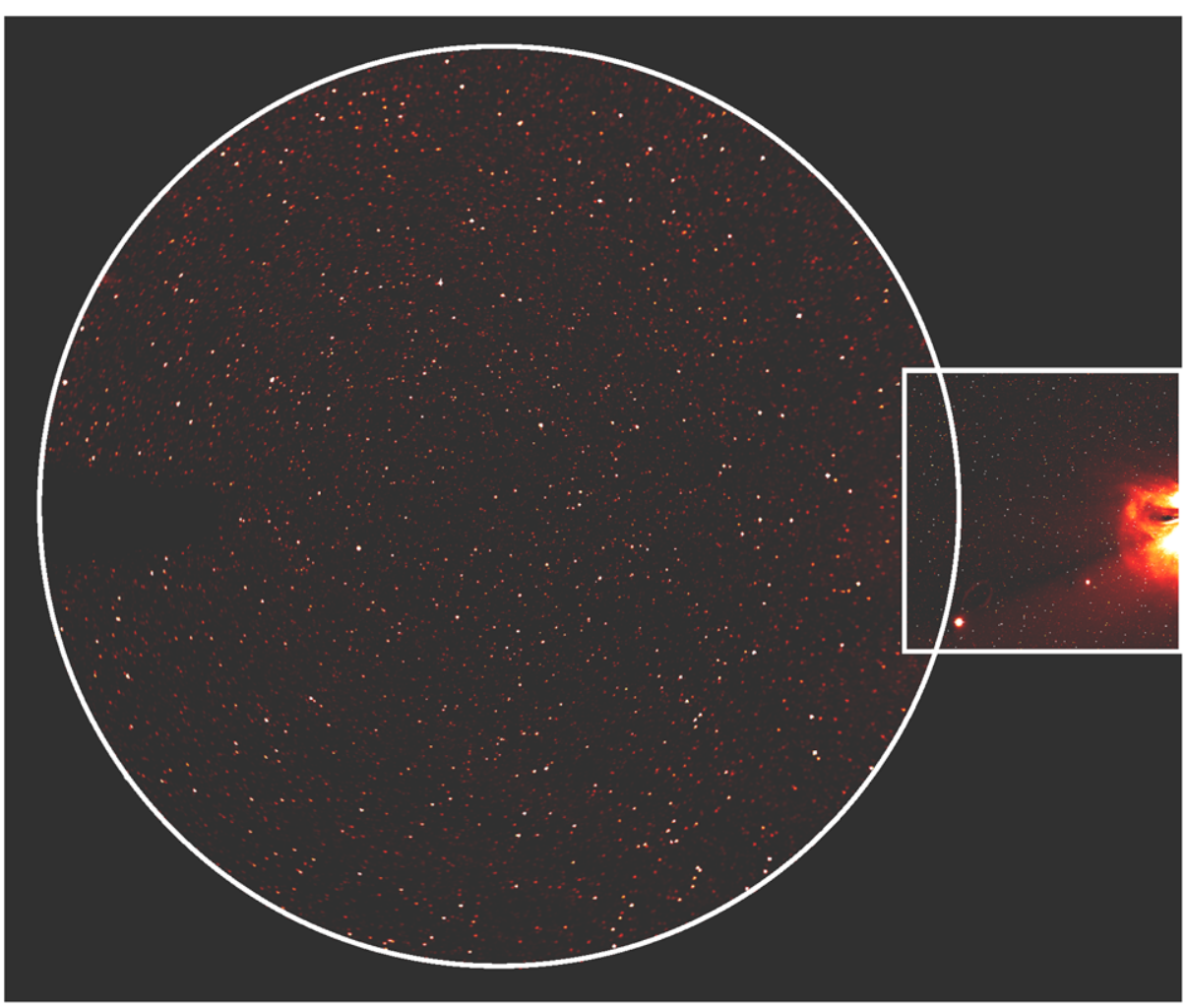

Figure 5 A combined HI-1 and HI-2 image from STEREO A on 25 January 2007.

\section{The CME of 24-25 January 2007}

We now describe the $24-25$ January 2007 events in greater detail, in particular because this is one of the first CMEs detected using the HI instruments. In this phase of the mission, the spacecraft were still very close to the Earth so the viewing locations are effectively from just outside the Sun -Earth line; later images with spacecraft - Sun - Earth angles of several tens of degrees and greater will have a far better view of the Sun-Earth line and Earth-directed CMEs. Nevertheless, these images demonstrate that the HI instrument is perfectly able to detect CMEs in the heliosphere and in that sense this observation is a significant landmark in space weather physics.

A snapshot of the CME, as seen in HI-1A and HI-2A, is shown in Figures 3 and 6. A full sequence of HI-1 A images is shown in Figure 7. The clarity of the images is best seen from the larger frames, but the sequence in Figure 7 shows the CME evolution.

The CME is propagating from the solar east limb. The sequence of frames starts at 18:01 UT on 24 January (top left). The frame at 22:01 UT shows a clear CME loop, some 67 degrees across, and extending into the HI- 1 field of view by about 3 degrees. In terms of position angle spread, subtended at the Sun, the CME appears to be approximately 70 heliographic degrees across. The same loop top at 02:01 UT on January 25 has decreased in intensity significantly and has extended into the field of view by some 6 degrees, that is, some 10 degrees (40 solar radii) from Sun centre. The same loop can be seen approaching the centre of the HI-1A field of view at 04:01 UT. 
Figure 6 The 25 January 2007 $\mathrm{CME}$ in the HI-2 field. The multiple-loop structures of the CME can be seen in the right-hand side of the frame. The striations in the lower half of the frame are due to the huge structured tail of Comet McNaught, which passed through the field of view earlier (see Fulle et al., 2007). The feature in the left-hand centre of the frame is an occulter designed to block off the Earth in the earliest stages of the mission.

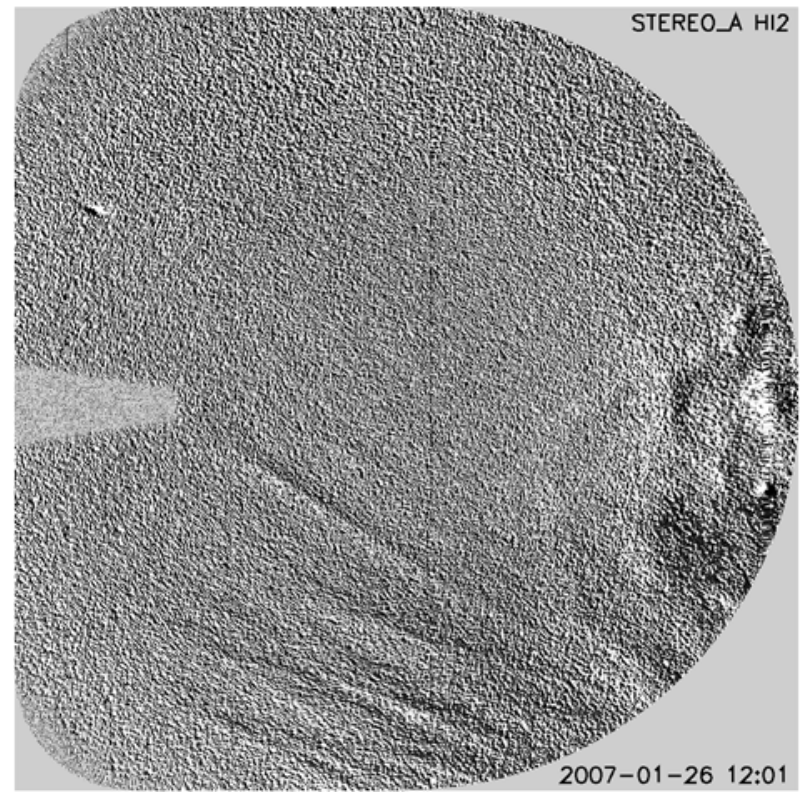

Figure 8 shows the geometry of the observation. At this early stage of the mission, the spacecraft (A) is rolled by 22.4 degrees from solar north (i.e., 17.6 degrees from ecliptic north). The spacecraft is Sun-pointed, and this is a roll about the axis directed towards the Sun. HI is mounted on the side of the STEREO spacecraft and with a field of view directed at an angle with respect to the spacecraft bore site; this converts into a translation and rotation of the field of view. For these observations this results in an effective roll of 21.7 degrees and a translation of 5.4 degrees, and we show this in the image by including the line XY, which is the location of the solar equator; the line $\mathrm{XZ}$ is the axis of the $\mathrm{HI}$ instruments on spacecraft A.

The Sun is located at $X$ in Figure 8 and is shown to scale. The image taken at 22:01 UT on 24 January is included in the figure. Two components to the CME are identified, namely A, a bright loop structure that appears to propagate along the path $\mathrm{XZ}$, and the more diffuse component whose front is denoted by $\mathrm{B}$, which appears to propagate outwards just north of the line XY. The time-height parameters for these two loop fronts are given in Table 2 and plotted in Figure 9. The distances are, of course, simply measured in the plane of the sky. The first observation, at 18:01 UT, does not show the CME; the data point is an approximation to the location of the outer front of the CME denoted by the near-Sun edge of the field of view. The salient point to note from these data is the fact that we are viewing a CME clearly, with identifiable loop structure, out to about 50 solar radii, over $0.23 \mathrm{AU}$, from Sun centre in these HI-1A images.

The time-altitude profiles of the CME fronts are shown in Figure 9. The ordinate is the distance from the solar limb. The measurement error on the location of the loop front detected in HI data may be of order 1.8 million $\mathrm{km}$, shown as error bars on the plot, which represents between $6 \%$ and $17 \%$ error on the distance to the solar limb. This estimate is simply a measurement based on the definition of the outer features of the CME structure but, in any case, it makes very little difference to the basic conclusions of these data. The HI-1A data points suggest that the CME front A is travelling at $551 \mathrm{~km} \mathrm{~s}^{-1}$ and front B is travelling at $604 \mathrm{~km} \mathrm{~s}^{-1}$. This is the velocity in the plane of the sky. 


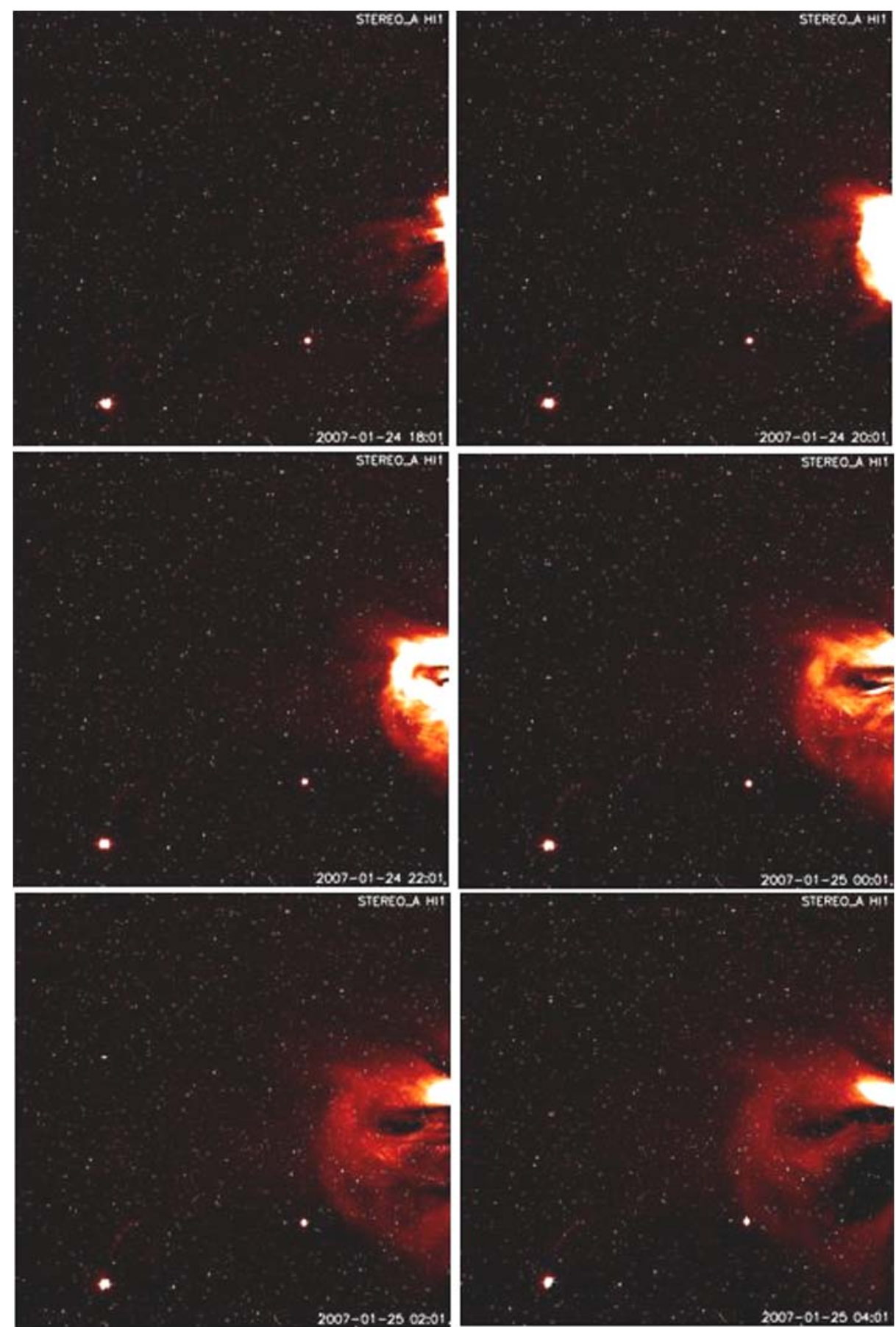

Figure 7 The 24-25 January CME in the HI-1A field of view. The time sequence runs from top left to bottom right; the image times and dates are given on each frame. 
Figure 8 The geometrical configuration of the observations (see text for explanation).

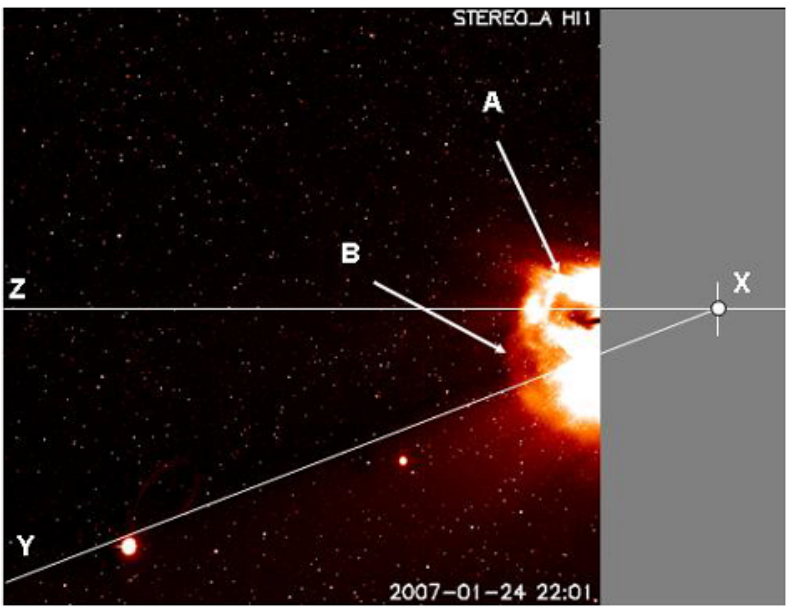

Table 2 The time - altitude parameters of the CME loop fronts A and B, measured along position angles of 68 and 83 degrees, respectively.

\begin{tabular}{|c|c|c|c|c|}
\hline \multirow{2}{*}{ Time (UT) } & \multicolumn{2}{|c|}{ Distance of front A from solar limb } & \multicolumn{2}{|c|}{ Distance of front B from solar limb } \\
\hline & Solar radii & Degrees & Solar radii & Degrees \\
\hline 18:01 24 Jan. & 16.8 & 4.2 & 17.4 & 4.4 \\
\hline 20:01 & 22.4 & 5.6 & 23.7 & 5.9 \\
\hline $22: 01$ & 26.9 & 6.7 & 30.1 & 7.5 \\
\hline 00:01 25 Jan. & 31.6 & 7.9 & 34.4 & 8.6 \\
\hline 02:01 & 38.9 & 9.7 & 42.4 & 10.6 \\
\hline 04:01 & 45.5 & 11.4 & 48.7 & 12.1 \\
\hline
\end{tabular}

We compare these observations with coronagraph data to (a) compare time-height analyses of particular features to establish the CME onset and propagation and (b) assess how the basic structure of the CME evolves as it passes through the inner heliosphere. For this, we use observations from the SOHO/LASCO coronagraph (Brueckner et al., 1995).

With regard to the time - height curve, data for the CME fronts in $\mathrm{C} 2, \mathrm{C} 3$, and HI-1A are all plotted in Figure 9, spanning a period of 15 hours and covering 50 solar radii, or 0.23 AU. Such coverage is unique.

As we shall see from a comparison of the images, these instruments are all detecting the same CME event. However, the precise CME feature used to produce the time-height curves from three different instruments may lead to a slight inconsistency among data points. Nevertheless, it can be concluded from Figure 9 that the CME is travelling at a near-constant velocity over about 50 solar radii, for a period of some 15 hours. The $\mathrm{C} 2$ measurements suggest a constant speed of $623 \mathrm{~km} \mathrm{~s}^{-1}$. Back-projecting this to the solar limb suggests a CME onset at 13:42 UT. The C3 data suggest a slightly slower speed of $580 \mathrm{~km} \mathrm{~s}^{-1}$. These values were derived using the CME front at a position angle of 90 degrees (directly over the east limb). Inspection of the images suggests that this is the "tongue" of bright material labelled B in Figure 8. The time-height curve for the top of the loop on the northern side of this structure, directed along $\mathrm{XZ}$ and labelled $\mathrm{A}$ in the figure, which is also a clear feature of the LASCO images, is denoted by asterisks in Figure 9. 
Figure 9 The time-altitude profile of the 24-25 January CME. The HI-1A fronts labelled $\mathrm{A}$ and $\mathrm{B}$ are denoted by the asterisk and box, respectively. The triangles and diamonds are the data points from the SOHO/LASCO C3 and C2 data, respectively.

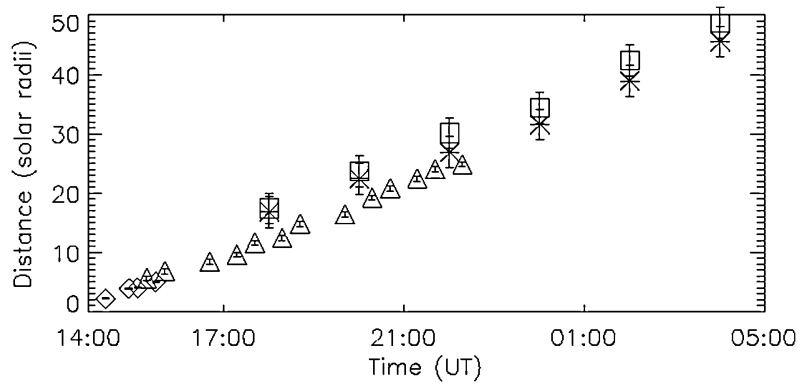

However, our principal interest is the outer edge and its onset. Thus, the LASCO velocities of $623 \mathrm{~km} \mathrm{~s}^{-1}$ in the $\mathrm{C} 2$ region and $580 \mathrm{~km} \mathrm{~s}^{-1}$ in the $\mathrm{C} 3$ field of view must be compared to the value of $604 \mathrm{~km} \mathrm{~s}^{-1}$ for the same feature in the HI-1A data. These values, and the errors associated with the positional measurements, would suggest that the CME is travelling at a constant velocity through much of the combined field, perhaps with early acceleration. This CME is almost certainly travelling faster than the ambient solar wind, yet the early deceleration (at less than $0.1 \mathrm{AU}$ ), which would have been suggested by the empirical models and projections just discussed, and any further more gradual deceleration, are not seen. We do point out that these data are from early observations; later sequences have much more coverage and would be better suited to detailed velocity profiles.

The other point to note from these HI-1A frames is well illustrated by the imaging of the ICME with planets in the same field of view. If we can show that any particular event is passing over Venus or Mercury for example, either using the two STEREO views or by combining available in situ data with the HI data, we can quite clearly see which portion of the expanding CME structure passes over the in situ instrumentation. The same will apply to Earth. Thus, these images illustrate the potential power of the HI system in interpreting the ICME signatures at Earth or other locations in the Solar System.

Figure 10 shows the CME as detected in the C2 component of LASCO. The basic CME structure shown in this image is remarkably similar to the structure of the CME shown in the HI image at, say, 04:01 UT. Both show evidence for a multiple loop structure with a very bright northern leg; even the southward "kink" in the southern leg of the CME is evident in both the HI and LASCO data. The outer loop, followed by the cavity structure, is certainly retained to at least 50 solar radii above the limb. Note that the $\mathrm{C} 2$ frame is shown with solar north directed upwards (i.e., it is rotated by some 21.7 degrees relative to the HI data); the line XY in Figure 8 would be horizontal in the $\mathrm{C} 2$ image. All of the LASCO images are oriented in the same way.

Figure 11 shows the same CME in the outer, C3, coronagraph from LASCO. The outer edge of the $\mathrm{C} 3$ field of view is 30 solar radii so there is considerable overlap with HI-1A. Not surprisingly, the same basic structure is clearly identifiable.

Comparing the physical characteristics of the same event detected at just a few solar radii and at approaching 50 solar radii is not simply an academic exercise. The self-similar expansion of a CME through the LASCO fields of view is well established but, as already discussed, the comparisons of near-Sun to near-Earth CMEs and ICMEs is far from straightforward and the evolution of CME structure as it passes through the heliosphere is an important issue to address. How do CMEs retain their initial structure during the passage from 0.1 to $1 \mathrm{AU}$ ? The images here show that, for this event, the basic structure is well preserved for almost 15 hours at least. Unfortunately, the last image of Figure 7 was the last HI-1A image for some hours so, whilst this early event illustrates a capability to address this issue fully, we lack some key images in this particular sequence. 
Figure 10 The same CME detected using the $\mathrm{SOHO} / \mathrm{LASCO}$ instrument in the $\mathrm{C} 2$ coronagraph. The edge of the field of view is 3 degrees from Sun centre.

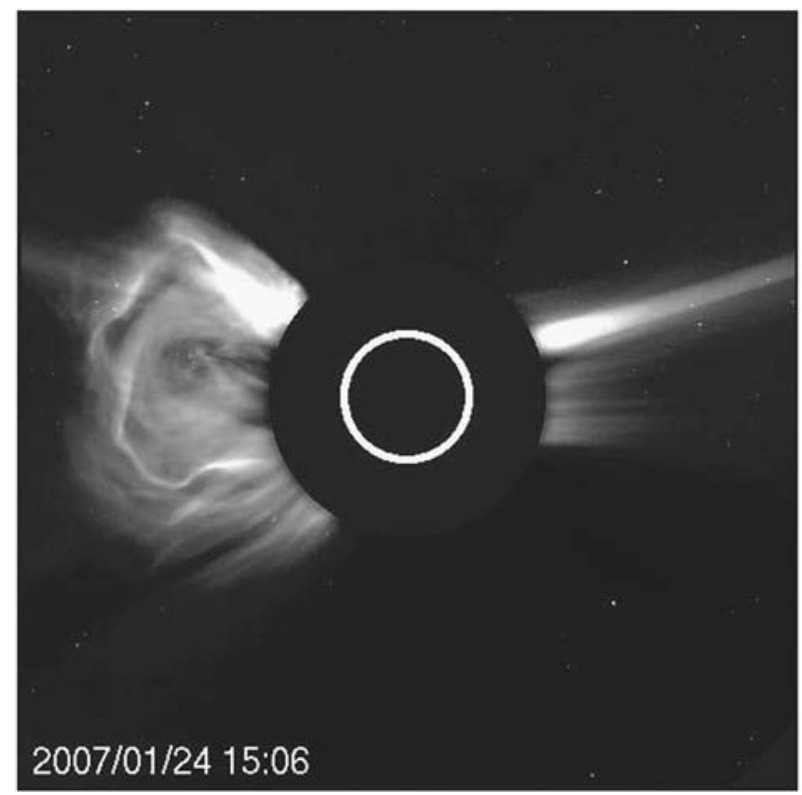

The projected onset time at 13:42 UT assumes a limb source region and no early, lowaltitude acceleration. Thus, we would suggest that it must be considered to be an upper limit; the CME onset was most likely earlier than this.

The onset appears to be associated in time with a B9 X-ray flare seen in the $1-8 \AA$ GOES data. The flare onset is given by the NOAA Space Environment Center as 13:45 UT, peaking at 14:52 UT and lasting for many hours. This event was not associated with a reported Halpha flare so we may suppose that it is near or beyond the limb. Thus, it could be on the eastern limb. Indeed, on 24 January, there was no reported active region on the solar disc in the eastern hemisphere but region 10940 rotated into view at S06 E77 on 26 January. This region is almost certainly associated with the CME activity and may have been the source of the X-ray flare.

We now examine the intensity of the CME, which is a useful indicator of the HI instrument performance as well as a good comparison to pre-launch image simulation analyses. In Figure 8 we reproduced one of the CME images. We examine the CME intensity along the line denoted XZ.

Figure 12a shows the intensity (in arbitrary units) along the line, plotted against image pixel number (from an image array 1024 pixels across) prior to the F-coronal subtraction; the curve is dominated by the F-corona and can be compared to the predicted F-coronal intensity of Koutchmy and Lamy (1985) (see Figure 2). Their Figure 3 suggests that above the solar equator the F-coronal intensity should be $8 \times 10^{-11} B_{\mathrm{o}}$ at the inner edge of HI-1 and $1 \times 10^{-12} B_{\mathrm{o}}$ at the outer edge. They suggest that these figures can be reduced by a factor of 2 over the polar regions.

A detailed analysis of the F-coronal intensity distribution is the subject of another paper in preparation. However, the intensity units plotted in Figure 12 are data units and we can briefly examine a preliminary conversion to physical units. Each image is a set of 25 exposures of $24 \mathrm{~s}$ each (i.e., a total of $600 \mathrm{~s}$ ). A preliminary conversion to units of solar brightness gives 1 data unit per image pixel (not CCD pixel) is equal to $5 \times 10^{-14} B_{0}$, where $B_{0}$ is the solar brightness. So, we must divide the intensities in Figure 12 by 600 and multiply by 5 

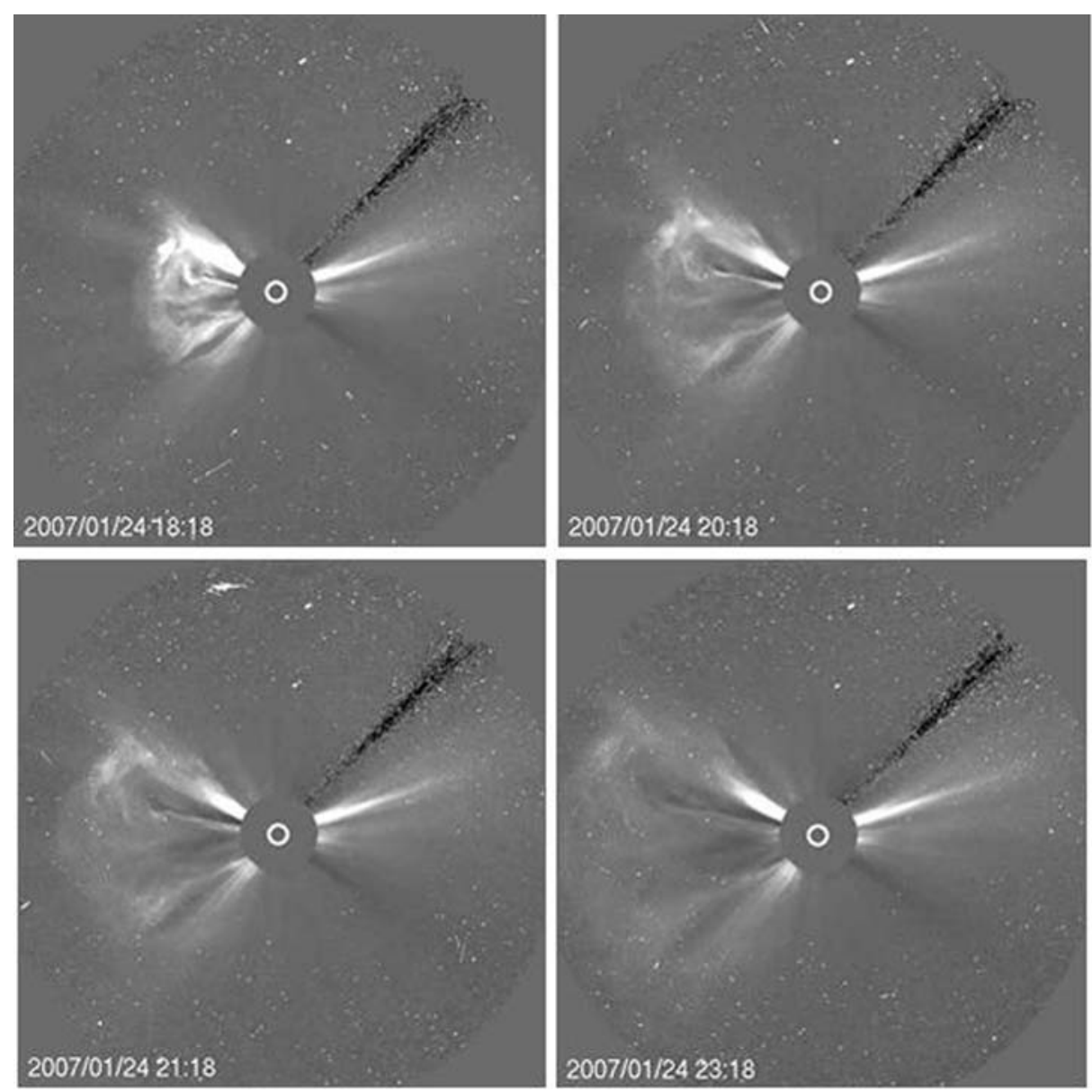

Figure 11 LASCO C3 observations of the 24 January CME.

$\times 10^{-14}$ to compare to solar brightness, as given in Figure 2. For Figure 12a, which means for the F-coronal intensity, we see the intensity drop from $1.25 \times 10^{-11}$ to $1.7 \times 10^{-13} B_{0}$.

These figures compare favourably with the values predicted by Koutchmy and Lamy (1985) when one considers that (a) the spacecraft was rolled by 22 degrees, so we are probably seeing reduced intensities relative to the equatorial values, and (b) the Koutchmy and Lamy values are from a modelled corona rather than measurement. Indeed, the latter fact stresses the need for the HI instruments to produce the first and definitive study of the measured F-coronal intensity along the Sun - Earth line.

Our aim here is to compare the CME intensity with that of the F-corona. We do this by removing the calculated F-coronal intensity. As mentioned, this F-coronal contribution has been subtracted from all of the images shown earlier. The intensity distribution, taken along the same line as for Figure 12a, with the F-corona subtracted, is shown in Figure 12b.

Figure $12 \mathrm{~b}$ shows some small-scale spiky structure caused by the presence of stars in the HI-A field of view, but it clearly reveals the intensity of the outer loop of the CME to peak at 900 counts, though it is clear that CME material is distinguishable from noise and stellar background levels at intensities of order 200 counts. At the same location (image 


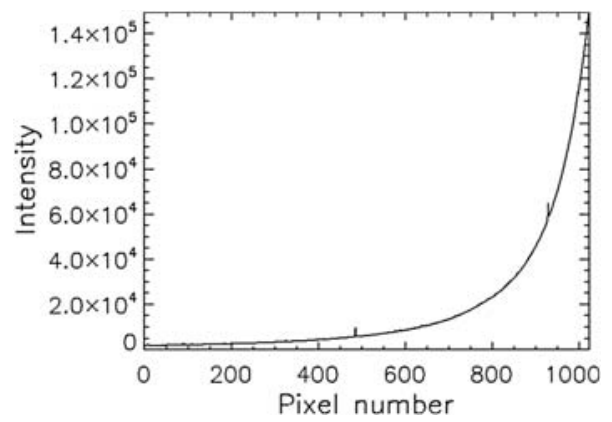

(a)

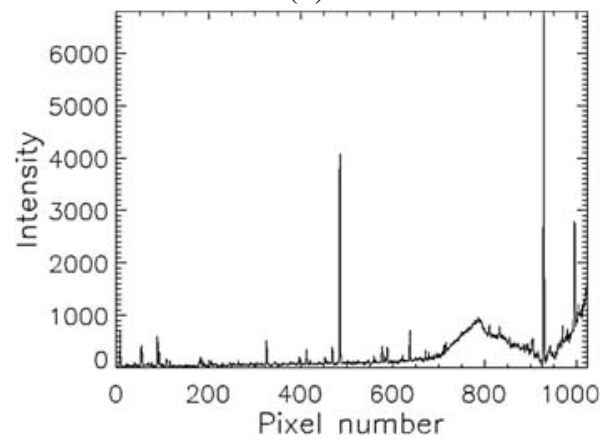

(b)

Figure 12 (a) The raw intensity (arbitrary data units) plotted against image pixel number across the HI field. (b) The same plot with the F-coronal intensity removed.

pixel) as the peak CME intensity, the total intensity, dominated by the F-corona, is of order 20000 counts. Thus, the CME to F-coronal intensity ratio is approximately 0.045 , and we can expect to detect readily CME intensities down to levels of about 0.01 of the F-coronal intensity. These measurements are made when the CME front is at about 40 solar radii. The estimated CME versus F-coronal intensity shown in Figure 2 and discussed in past papers (see Harrison, Davis, and Eyles, 2005) suggests that at this location we expect a CME to F-coronal intensity of 0.02. In terms of solar intensity, using the details given here, the CME front has a peak intensity of $7.5 \times 10^{-14} B_{0}$ and the data suggest that we may detect CME features to values of $1.7 \times 10^{-14} B_{0}$, at these distances from the Sun. This in itself is a remarkable achievement.

The important issue here is the fact that we are able to demonstrate that we have readily detected CME material down to the order of one-hundredth of the F-coronal intensity. In this particular case we see a CME loop that has a maximum intensity about one-twentieth that of the F-corona. These figures confirm that the HI instrument is capable of detecting CMEs of the intensities anticipated.

An important feature of the HI-1A CME observations is shown in Figure 13. The figure shows a stack of the five partial frames containing the CMEs from 24-25 January. Two white guidelines are drawn to aid the eye. However, two features of the data are obvious. First, the footpoints do not move apart even though the sunward edge of the frame is 15 solar radii from the solar limb. This is in keeping with traditional coronagraph findings (i.e., for any given distance as the CME expands outwards, the CME width or footpoint separation remains fairly fixed). In addition, despite intensity variations, the physical extent of each 
Figure 13 A stack of the five CME frames from Figure 7 showing the relative footpoint locations and physical extent.

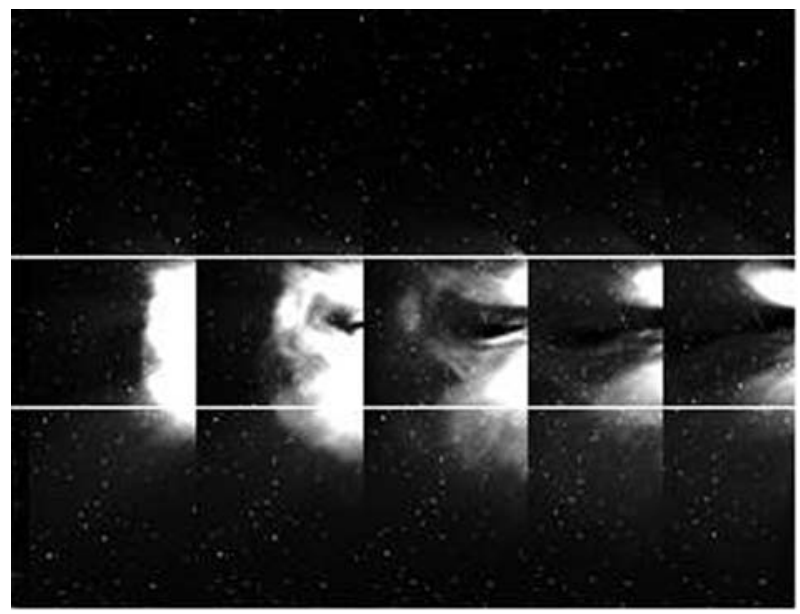

footpoint remains fairly constant. Remember that these observations are made over a period of tens of hours. This is a trivial observation but an important result, which is in complete agreement with coronagraph observations. However, it is an important point to note because many CME interpretations, especially those relating to space weather, and some interpretations of the links between CMEs and coronal waves imply that the nonradial expansion of CMEs is significant. Yet even to these distances from the Sun, non-radial expansion is clearly not evident.

A key observation of interest with regard to the HI observations is the ability to detect CMEs in the HI-2 frames. We have demonstrated the ability to detect CMEs in the inner, HI-1, field of view but to detect CMEs out to elongation angles consistent with the location of the Earth is extremely important. For this CME event sequence, we show a series of HI2A frames in Figure 14 that indeed show detection of the CME into this outer field of view. One of the frames was shown in a larger format in Figure 6.

The CME is fading rapidly as it crosses the HI-2A field of view. The images show that the CME is detected to over 30 degrees from the solar limb (a quarter of the field) but there do appear to be "forerunner" structures visible out to 42 degrees.

The brightest loop appears to be propagating along the axis of the field of view, which appears to relate, spatially, to the event in HI-1 labelled A. We tabulate the altitude history of this event in HI-1A and HI-2A in Table 3 and plot this in Figure 15. The HI-1A data match well to the $551 \mathrm{~km} \mathrm{~s}^{-1}$ line (dotted line). If the HI-2A loop is indeed the same feature the data set does suggest some deceleration at distances of over tens of degrees from the Sun. This deceleration is consistent with the numerical study of Gonzalez-Esparza et al. (2003). However, we would advise caution. This is the first event that has been tracked in this way and we need more observations of ICMEs at such distances from the Sun before we can draw definitive conclusions. The image cadence in this particular data set was such that there was a large observational gap between 11 and 25 degrees and a more complete tracking of the event in this phase would have (a) produced a better time-altitude profile and (b) allowed more confidence in linking particular features in the HI-1 A and HI-2A data. Nevertheless, these data do show a capability to track a CME from the vicinity of the Sun to tens of degrees and the fact that a deceleration is seen tens of degrees from the Sun, after a long phase of constant velocity, is a new observational result.

If we assume that the CME is travelling at 90 degrees to the spacecraft - Sun line, then the loop-top profile translates to a tracked distance of 95 million $\mathrm{km}$ (32.5 degrees). In addition, 

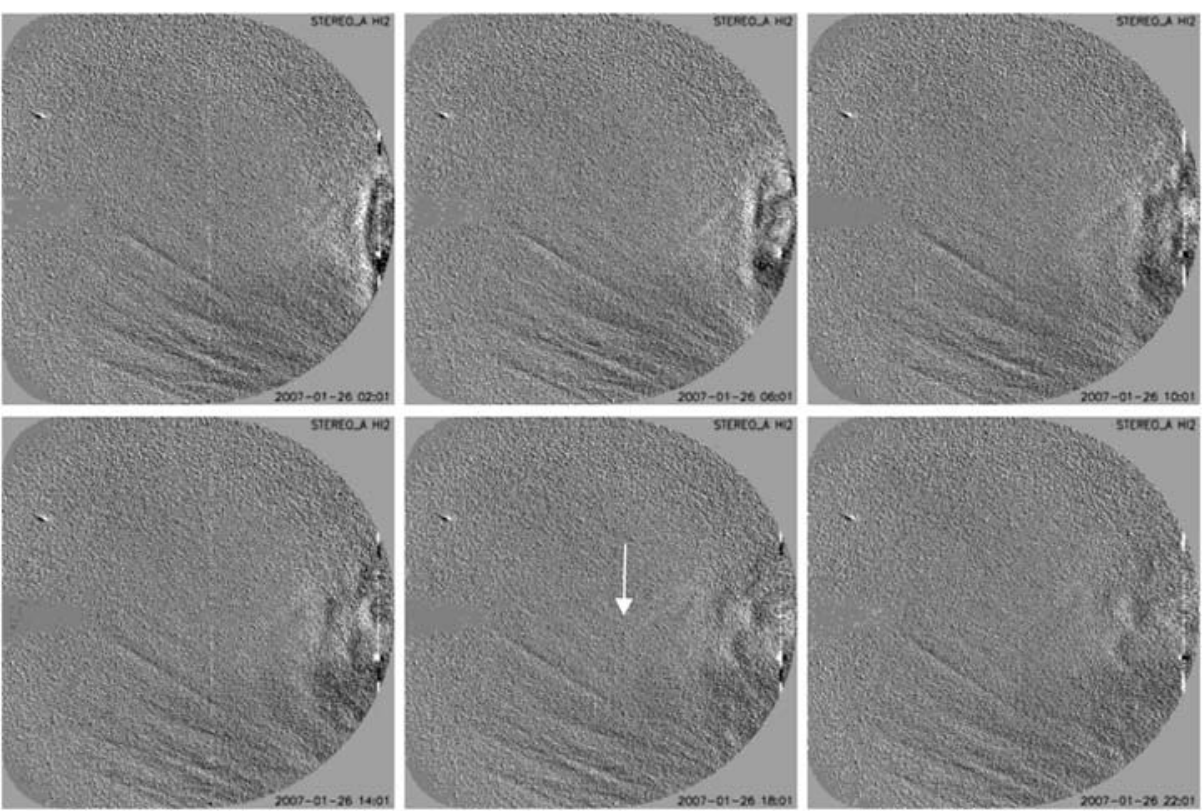

Figure 14 A series of six differenced HI-2A frames from 26 January 02:01 to 22:01 UT showing the same CME as discussed in the text. Whilst the main CME structure is clearly visible, there are ghostly forerunner structures - indicated in one image by the white arrow.

Table 3 The time - altitude parameters of the HI-1 and HI-2 CME loop fronts.

\begin{tabular}{lll}
\hline Time (UT) & Instrument & Distance of front A from solar limb (degrees) \\
\hline 18:01 24 Jan. & HI-1A & 4.2 \\
20:01 & HI-1A & 5.6 \\
22:01 & HI-1A & 6.7 \\
02:01 25 Jan. & HI-1A & 9.7 \\
04:01 & HI-1A & 11.4 \\
02:01 26 Jan. & HI-2A & 24.7 \\
06:01 & HI-2A & 27.0 \\
10:01 & HI-2A & 29.8 \\
14:01 & HI-2A & 30.9 \\
18:01 & HI-2A & 32.5 \\
\hline
\end{tabular}

if we believe that the "forerunner" structures are part of this event (Figure 14), detected to 42 degrees, this translates to 135 million $\mathrm{km}$ from the limb. Tracking a CME over such distances from the Sun, with the potential for such tracking along the Sun-Earth line, is a significant advance over previous capabilities.

We should add that the image processing and background subtraction techniques are being developed actively in this early phase of the mission and later analysis may well reveal clearer structure in the later frames well into the HI-2 field of view. Nevertheless, at 
Figure 15 The time-altitude history of loop A as detected in HI-1A and HI-2A showing a capability for tracking CMEs to over 30 degrees.

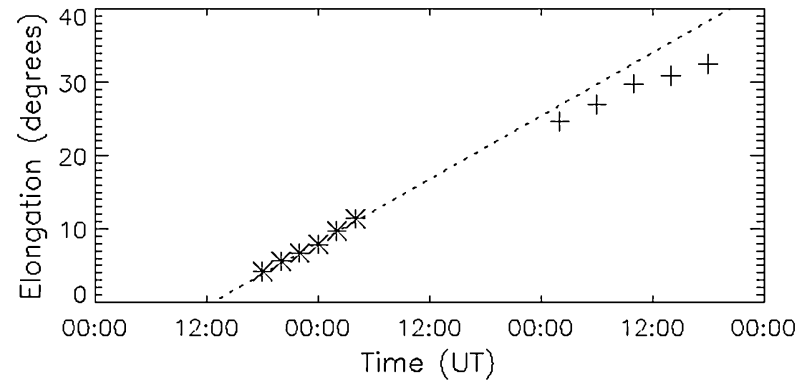

this early phase in the STEREO operation, it is important to announce that the ability to detect CMEs in both $\mathrm{HI}$ instruments has been well demonstrated.

The forerunner may well be an example of a separate erupting magnetic structure that contains little excess mass. Its eruption from the corona is triggered around the same time as the main CME (in this case slightly earlier) but it is otherwise a distinct entity.

We point out that the striations in the lower portions of the images of Figure 14 are structure from the McNaught comet tail. The coma of McNaught passed through the HI field of view in mid January (see Fulle et al., 2007) and the aftermath of an extended tail can be detected quite clearly for some weeks after the event.

At the time of the 24-25 January observations, the spacecraft separation angles (spacecraft-Sun-Earth) were, in fact, only 0.15 and 0.39 degrees, for the B and A spacecraft, respectively, giving a spacecraft - Sun - spacecraft separation of 0.54 degrees. Given these locations there is no significant field of view overlap between HI-A and HI-B at this time. For later events we may see particular CME events from two angles simultaneously. Thus, the HI-A data for the 24-25 January events makes no contribution to the analysis here.

\section{Other CME Events}

We have really focused on one event sequence early in the operations phase of the STEREO mission. Recognising that the particular event discussed was detected early in the mission and with a narrow spacecraft - Sun - Earth angle, we complement this here with some observations of other CME events detected in the March - April period, with larger separation angles.

The events, shown in Figure 16, are for 6 March, 12 March, 21 March, and 20 April 2007. They are all shown in HI-1 images from spacecraft A, which is at a spacecraft - Sun - Earth angle of 1.2, 1.4, 1.8, and 3.4 degrees, respectively. Even though the latest event shown has a spacecraft - Sun - Earth angle about six times that of the 24-25 January observation period, the geometry is such that the observation is barely outside the Sun-Earth line in reality. However, to put that in perspective, at the time of the 20 April observation, spacecraft $\mathrm{A}$ is about 9 million $\mathrm{km}$ ahead of the Earth. Given these small angles, for Earth-directed CMEs the events would be detected even in the HI data as clouds with extremely broad position angle spreads, the equivalent of the halo CMEs seen in coronagraph data.

The events discussed so far have been from observations using the HI-A instrument. Figure 17 shows an example of an HI-1B observation of a CME on 1 April 2007. Unlike the HI-A images, the Sun is 4 degrees off the left-hand side of this image, rather than the right-hand side. The CME is a well-defined event on the left side of the frame, which is displayed as a differenced image. The oval artefact dominating the right-hand side of the 

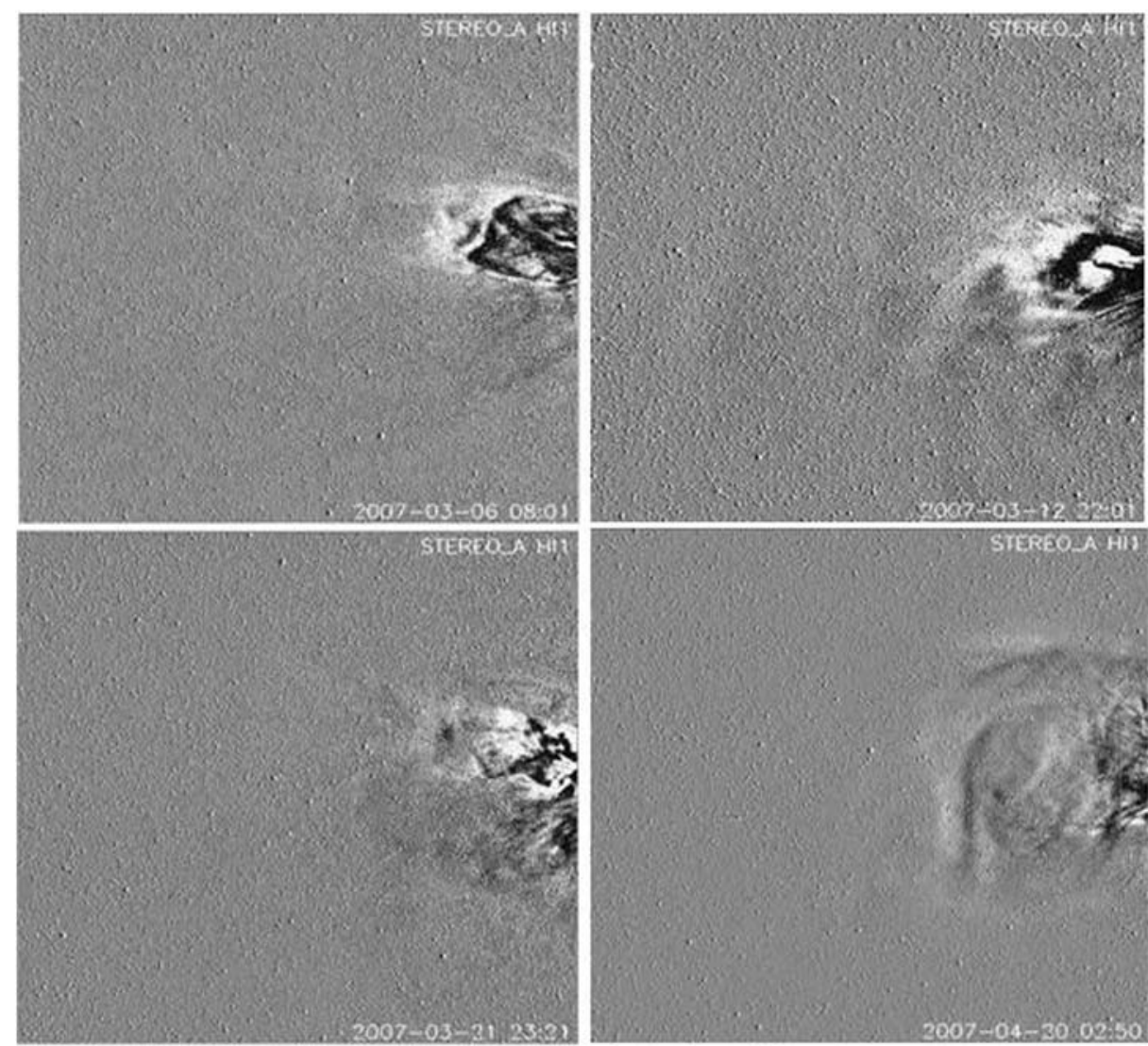

STER2OYA Hils

Figure 16 A selection of CMEs detected during March and April 2007 shown as differenced images in the HI-1A data.

frame is an optical ghost generated because, at this stage of the mission, the Earth is within the HI-1B field of view. Indeed, the white vertical bar near the right edge of the frame shows saturation at the location of the Earth. Despite the fact that at this early phase the Earth is in the instrument field of view it is clear that CME observations are being made successfully. As the Earth recedes from the spacecraft, these severe artefacts will diminish until the Earth is viewed in the same way as Venus and Mercury are seen in the images shown in Figures 3, 4, and 7. It must be noted that the detection of such a bright image of the Earth is a fact of life for the "behind" spacecraft as it pulls back into an orbit just outside the Earth's in the early mission.

The events shown here are excellent examples of the performance of the $\mathrm{HI}$ instruments and such an observation from a Sun-orbiting spacecraft out of the Sun-Earth line is a breakthrough. However, to witness CME events with spacecraft -Sun-Earth angles in excess of, say, 20 degrees, we must wait until November 2007. 
Figure 17 A CME detected in the HI-B instrument on 1 April 2007. See text for explanation.

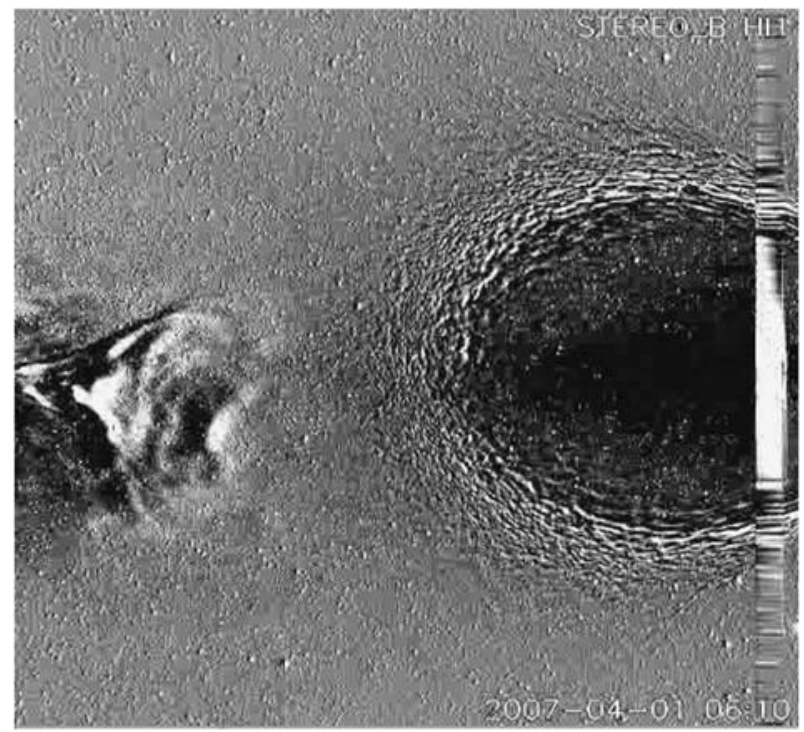

\section{Discussion and Conclusions}

It was the purpose of this study to demonstrate that the Heliospheric Imagers aboard the STEREO spacecraft can detect CMEs in the heliosphere (i.e., ICMEs), demonstrating a capability for tracking CMEs from a vantage point outside the Sun-Earth line. This has been done, mainly through the analysis of one event and with brief illustrations of a handful of other events. In doing so, we have shown an ability to detect and track events from the vicinity of the Sun out to the vicinity of the Earth.

This first announcement heralds the start of detailed analyses of the physics of CMEs in the heliosphere using HI data and, later in the mission, the clear detection, tracking, and analysis of CMEs as they propagate along the Sun - Earth line. Investigation of the images shown also reveals some useful aspects regarding CME propagation into the heliosphere. We touch on these here.

The 24-25 January CME is well observed in the HI and SOHO/LASCO data and is effectively tracked from the corona to over 30 degrees, and possibly up to 42 degrees. Using LASCO and HI-1 we witness constant speeds of ascent at about $600 \mathrm{~km} \mathrm{~s}^{-1}$ over at least 15 hours, with evidence in LASCO C2 for acceleration at the lowest altitudes. However, linking the HI- 1 and HI-2 data suggests that we witness deceleration at distances of approximately 20 degrees and over.

The HI and LASCO images display a self-similar expansion of the January CME to at least 50 solar radii. Given the complexities of relating ICME and CME signatures, as mentioned here, clear observations of CME evolution during propagation in the heliosphere is essential. Thus, these observations display a significant capability for extending such knowledge. In keeping with such expansion, the 24-25 January CME observations clearly shows that the legs of the expanding CME remain fixed as the event progresses; there is no significant non-radial expansion of the CME legs even as the event propagates well into the heliosphere.

The HI-2A images also show a clear multiple loop structure, but at this stage in the analysis it is harder to compare the features to those nearer to the Sun. Indeed, the HI-2A 
images, whilst showing loop-like features containing structure, also appear to reveal diffuse, outer "forerunner" features extending towards the middle of the field of view.

Overall, we have been successful in tracking the ejection of 24-25 January in the HI instruments from 4 to over 30 degrees, and possibly to over 40 degrees in this initial analysis.

Examination of the intensities of the F-corona and the CME in question demonstrates that the HI instruments are well able to detect CMEs of order 100 times weaker in intensity than the F-corona; for the particular CME in question, the loop intensity was about one-twentieth that of the F-coronal intensity.

Preliminary analysis indicates that we detect F-coronal intensities ranging from 1.25 $\times 10^{-11}$ to $1.7 \times 10^{-13} B_{0}$ in the region from 4 to 24 degrees from the Sun (15 to 95 solar radii above the limb), which is consistent with but slightly lower than the intensity values predicted by Koutchmy and Lamy (1985). Extending such unique measurements to more observations is clearly essential for characterising the F-coronal distribution out to Earth-like distances.

To illustrate the HI instrument performance, we have demonstrated a capability of detecting CMEs of intensities down to $1.7 \times 10^{-14} B_{\mathrm{o}}$ using measurements on a CME front at 40 solar radii, where the F-corona is two orders of magnitude brighter than the CME intensity.

With these first CME observations made by the Heliospheric Imager on STEREO we are witnessing the beginning of a new era in CME research, the emphasis now moving into the tracking and development of CMEs in the heliosphere and, in particular, those directed towards and arriving at the Earth.

Acknowledgements The Heliospheric Imager (HI) instrument was developed by a collaboration that included the Rutherford Appleton Laboratory and the University of Birmingham, both in the United Kingdom, and the Centre Spatial de Liège (CSL), Belgium, and the US Naval Research Laboratory (NRL), Washington DC, USA. The STEREO/SECCHI project is an international consortium of the Naval Research Laboratory (USA), Lockheed Martin Solar and Astrophysics Lab (USA), NASA Goddard Space Flight Center (USA), Rutherford Appleton Laboratory (UK), University of Birmingham (UK), Max-Planck-Institut für Sonnensystemforschung (Germany), Centre Spatial de Liege (Belgium), Institut d'Optique Théorique et Appliquée (France), and Institut d'Astrophysique Spatiale (France). SOHO is a mission of international collaboration between ESA and NASA. LASCO was built and is operated by a consortium led by the US Naval Research Laboratory and including the Max-Planck-Institut für Sonnensystemforschung, Lindau, Germany, the School of Physics and Astronomy, the University of Birmingham, UK, and Laboratoire d'Astronomie Spatial, Marseille, France.

\section{References}

Brueckner, G.E., Howard, R.A., Koomen, M.J., Korendyke, C.M., Michels, D.J., Moses, D.J., Socker, D.G., Dere, K.P., Lamy, P.L., Llebaria, A., Bout, M.V., Schwenn, R., Simnett, G.M., Bedford, D.K., Eyles, C.J.: 1995, Solar Phys. 162, 357.

Davis, C.J., Harrison, R.A.: 2005, Adv. Space Res. 36, 1524.

Defise, J.-M., Halain, J.-P., Mazy, E., Rochus, P., Howard, R.A., Moses, J.D., Socker, D.G., Harrison, R.A., Simnett, G.M.: 2003, Proc. SPIE 4853, 12.

Eyles, C.J., Simnett, G.M., Cooke, M.P., Jackson, B.V., Buffington, A., Hick, P.P., Waltham, N.R., King, J.M., Anderson, P.A., Holladay, P.E.: 2003, Solar Phys. 217, 319.

Fulle, M., Leblanc, F., Harrison, R.A., Davis, C.J., Eyles, C.J., Halain, J.-P., Howard, R.A., Bockelee-Morvan, D., Cremonese, G., Scarmato, T.: 2007, Astrophys. J. Lett. 661, L93.

Gonzalez-Esparza, J.A., Lara, A., Perez-Tijerina, E., Santillan, A., Gopalswamy, N.: 2003, J. Geophys. Res. 108(A1), 1039.

Gopalswamy, N., Mewaldt, R., Torsti, J. (eds.): 2006, Solar Eruptions and Energetic Particles, AGU Geophys. Monograph 165, ISBN-13: 978-0-87590-430-6.

Gopalswamy, N., Lara, A., Lepping, R.P., Kaiser, M.L., Berdichevsky, D., St Cyr, O.C.: 2000, Geophys. Res. Lett. 27(2), 145. 
Gopalswamy, N., Lara, A., Yashiro, S., Kaiser, M.L., Howard, R.A.: 2001, J. Geophys. Res. 106(A12), 29207.

Harrison, R.A., Davis, C.J., Eyles, C.J.: 2005, Adv. Space Res. 36, 1512.

Howard, R.A., Moses, J.D., Socker, D.G.: 2000, Proc. SPIE 4139, 259.

Howard, R.A., Michels, D.J., Sheeley, N.R., Koomen, M.J.: 1982, Astrophys. J. Lett. 263, 101.

Jackson, B.V., Leinert, C.: 1985, J. Geophys. Res. 90, 10, 759.

Koutchmy, S., Lamy, P.: 1985, In: Giese, R.H., Lamy, P. (eds.) Properties and Interactions of the Interplanetary Dust, IAU Colloq. 85, Reidel, Dordrecht, 63. ISBN 90-277-2115-7.

Kunow, H., Crooker, N.U., Linker, J.A., Schwenn, R., von Steiger, R. (eds.): 2006, Coronal Mass Ejections, Space Sciences Ser. of ISSI 21, ISBN: 978-0-387-45086-5. Reprinted from Space Sci. Rev. 123.

Richter, I., Leinert, C., Planck, B.: 1982, Astron. Astrophys. 110, 115.

Riley, P., Schatzman, C., Cane, H.V., Richardson, I.G., Gopalswamy, N.: 2006, Astrophys. J. 647, 648.

Socker, D.G., Howard, R.A., Korendyke, C.M., Simnett, G.M., Webb, D.F.: 2000, Proc. SPIE 4139, 284.

Wimmer-Schweingruber, R.F., Crooker, N.U., Balogh, A., Bothmer, V., Forsyth, R.J., Gazis, P., Gosling, J.T., Horbury, T., Kilchenmann, A., Richardson, I.G., Richardson, J.D., Riley, P., Rodriguez, L., von Steiger, R., Wurz, P., Zurbuchen, T.H.: 2006, Space Sci. Rev. 123, 177. 